Takahashi, A. Studies on 3D Fusion Reactions in TiDx under Ion Beam Implantation. in Tenth International Conference on Cold Fusion. 2003. Cambridge, MA: LENR-CANR.org. This paper was presented at the 10th International Conference on Cold Fusion. It may be different from the version published by World Scientific, Inc (2003) in the official Proceedings of the conference.

\title{
Studies on 3D Fusion Reactions in TiDx under Ion Beam Implantation
}

\author{
A. Takahashi, H. Miyamaru, K. Ochiai, Y. Katayama, T. Hayashi and T. Dairaku \\ Osaka University, Yamadaoka 2-1, Suita, Osaka, Japan \\ E-mail: akito@nucl.eng.osaka-u.ac.jp
}

With certain conditions for target-samples and beams, we have identified specific particles (e.g., $4.75 \mathrm{MeV}$ tritons and ${ }^{3} \mathrm{He}-$ particles, and $15.9 \mathrm{MeV}$ deuterons) from 3D fusion reactions with yield ratios $[3 \mathrm{D}] /[2 \mathrm{D}]$ on the order of $1 \mathrm{E}-4$ to $1 \mathrm{E}-3$, in contrary to the calculated $[3 \mathrm{D}] /[2 \mathrm{D}]$ yield ratio of $1 \mathrm{E}-30$ according to conventional random nuclear reaction theory ${ }^{2,3)}$. The increasing trend of yield ratios in lower energy regions (less than $100 \mathrm{keV}$ for deuteron) ${ }^{4}$, suggests that the enhanced 3D reactions were not attributed to the direct reactions with incident d-beam, but to the indirect 3D fusion out of the slowing down range of the beam.

\section{Introduction}

This is a review of our experimental studies over the past twelve years in the detection of three-body (3D) deuteron fusion in TiDx samples using low energy ion beam irradiation.

If there exists a linkage between nuclear reaction (nuclear physics) and electron behaviors in condensed matter (condensed matter physics), a new class of nuclear reactions in condensed matter may be induced under certain conditions of lattice dynamics with low particle energy. Since 1991, we have studied possible occurrence of highly enhanced multi-body deuteron fusion in metal-deuteride samples under low energy deuteron beam irradiation, based on our speculation that the order of atoms and electrons under transient motion around lattice focal points should greatly enhance the three-body fusion process, compared with random nuclear reactions. ${ }^{1-3}$

Our experimental results suggest that there exists strong screening effect on the Coulomb repulsive force of $d-$ $\mathrm{d}$ interaction by transient "electronic quasi-particles", 6 and this greatly enhances 2D and 3D fusion reactions, and even $4 \mathrm{D}$ fusion reactions.

\section{2. ${ }^{3} \mathrm{He}+\mathrm{t}$ Branch}

To confirm this hypothesis, we have done a series of nuclear physics type experiments using low energy beams of deuterons and protons implanting into metal-deuteride targets ${ }^{1-4}$. We expected that by implanting low energy deuterons into highly deuterated (x.>1.8) metal like TiDx, conditions for deuteron cluster fusion in focal points of lattice might be somehow induced in the near region of slowing down path (range) of bombarded deuterons, where phonon excitations for trapped deuterons might be stimulated by the ionization and atom-recoil processes of ions as they slow down. Under these conditions of ion-beam implantation to metal deuteride, coherent motion of deuterons in metal is thought to be induced at the end of the beam scanning range or deeper, and multi-body fusion takes place ${ }^{3,4}$. The possible channels of the three-body fusion are assumed to be as follows:

$$
\begin{aligned}
\mathrm{D}+\mathrm{D}+\mathrm{D} \rightarrow{ }^{6} \mathrm{Li}^{*}(25.32 \mathrm{MeV}) & \rightarrow \mathrm{d}(15.9 \mathrm{MeV})+{ }^{4} \mathrm{He}(7.90 \mathrm{MeV}) \\
& \rightarrow \mathrm{t}(4.75 \mathrm{MeV})+{ }^{3} \mathrm{He}(4.75 \mathrm{MeV}) \\
& \rightarrow \mathrm{n}+\mathrm{p}+{ }^{4} \mathrm{He}+20.1 \mathrm{MeV} \\
\mathrm{H}+\mathrm{D}+\mathrm{D} \rightarrow{ }^{5} \mathrm{Li}^{*}(21.88 \mathrm{MeV}) & \rightarrow \mathrm{p}(19.1 \mathrm{MeV})+{ }^{4} \mathrm{He}(4.77 \mathrm{MeV}) \\
& \rightarrow \mathrm{d}(3.30 \mathrm{MeV})+{ }^{3} \mathrm{He}(2.20 \mathrm{MeV})
\end{aligned}
$$

We assume that the reaction channel (b) is unique, having two mass-three charged particles with same kinetic energies 4.75 MeV. This does not exist in known two-body reactions of deuterons with light elements (from proton to nitrogen-15), which might form contaminants, and possible impurity reactions with incident deuterons. Therefore, when we could detect a triton and ${ }^{3} \mathrm{He}$ with $4.75 \mathrm{MeV}$, we can be confident this is evidence of threebody deuteron fusion. By using a proton beam and a detecting channel (d), we are able to determine whether the cause of multi-body fusion can be attributed to the direct reaction between incident ion-beam and closelypacked d-d pairs transiently excited in target, or whether it can somehow be attributed to the indirect three-body $(\mathrm{D}+\mathrm{D}+\mathrm{D}$ induced by proton) fusion that is pure coherent fusion in condensed matter. 
We have tried to detect charged particles emitted form these channels (b) and (d) using silicon surface barrier detectors (SSBD), using the experimental system shown in Figure 1. We used beam energy of $300 \mathrm{keV}$ down to $50 \mathrm{keV}$ for $\mathrm{D}^{+}$or $\mathrm{H}^{+}$ions. We set up two detector systems. One was a counter-telescope spectrometer using $\mathrm{E}$ and $\Delta \mathrm{E}$ detectors of SSBDs, to identify particle-types and their initially emitted kinetic energies. The other was the so-called Ek detector which aimed to detect total the kinetic energies of emitted charged particles.

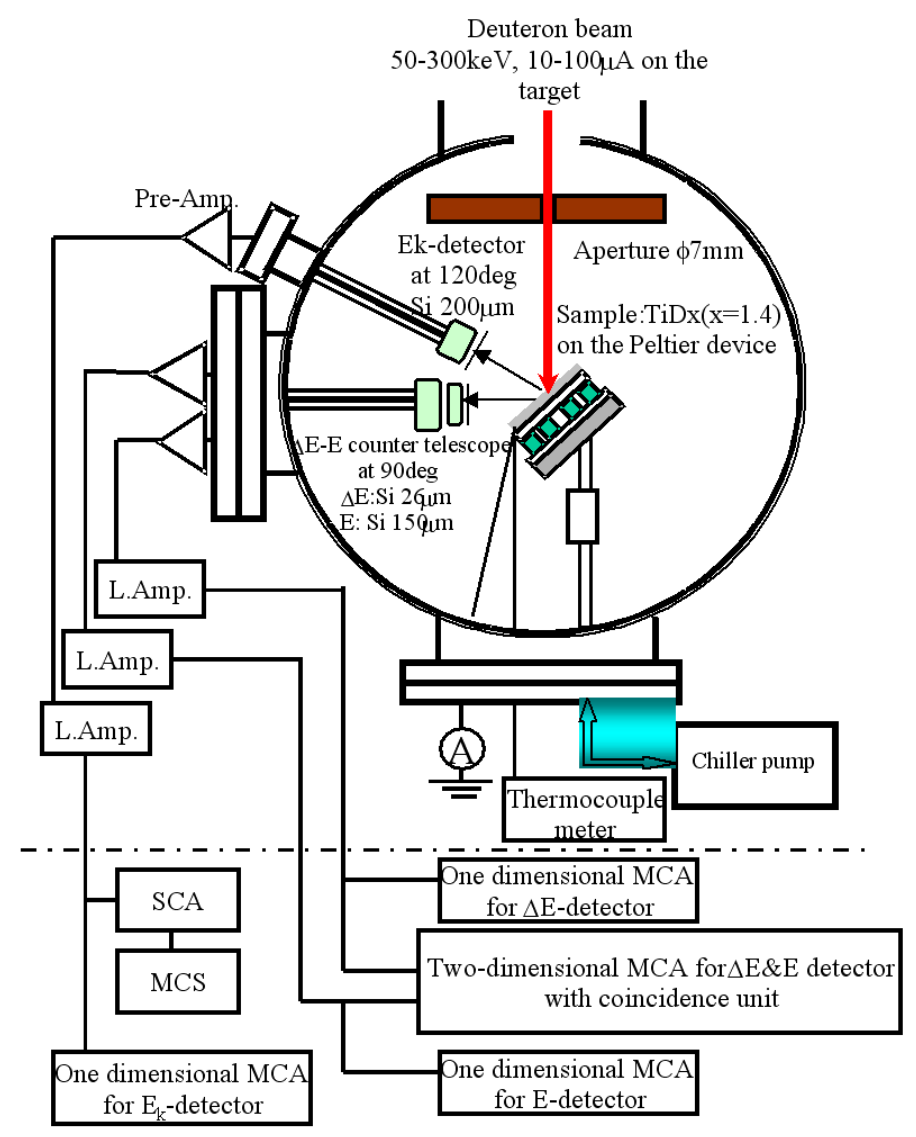

Figure 1. Experimental system for low energy $\mathrm{D}(\mathrm{H})$ beam implantation and emitted charged particle spectroscopy

In some of the previous experiments, we could not precisely search the unique charged particle spectrum of $4.75 \mathrm{MeV}$ triton by channel (b), because of pileup signals of D-D reactions. We introduced a pile-up removing technique based on wave-shape discrimination. ${ }^{4,7}$. In proton beam experiment, high energy protons from channel (d) having been observed in the $17-20 \mathrm{MeV}$ energy region. They have been detected with high reproducibility. To investigate whether multi-body fusion includes incident particles (direct multi-body fusion) or not (indirect multi-body fusion), we tried an experiment in which we irradiated $\mathrm{TiD}_{\mathrm{x}}$ target with Si-beam. We observed an isolated peak at about $3.6 \mathrm{MeV}$, which we suppose is the response of tritons by channel (b). 


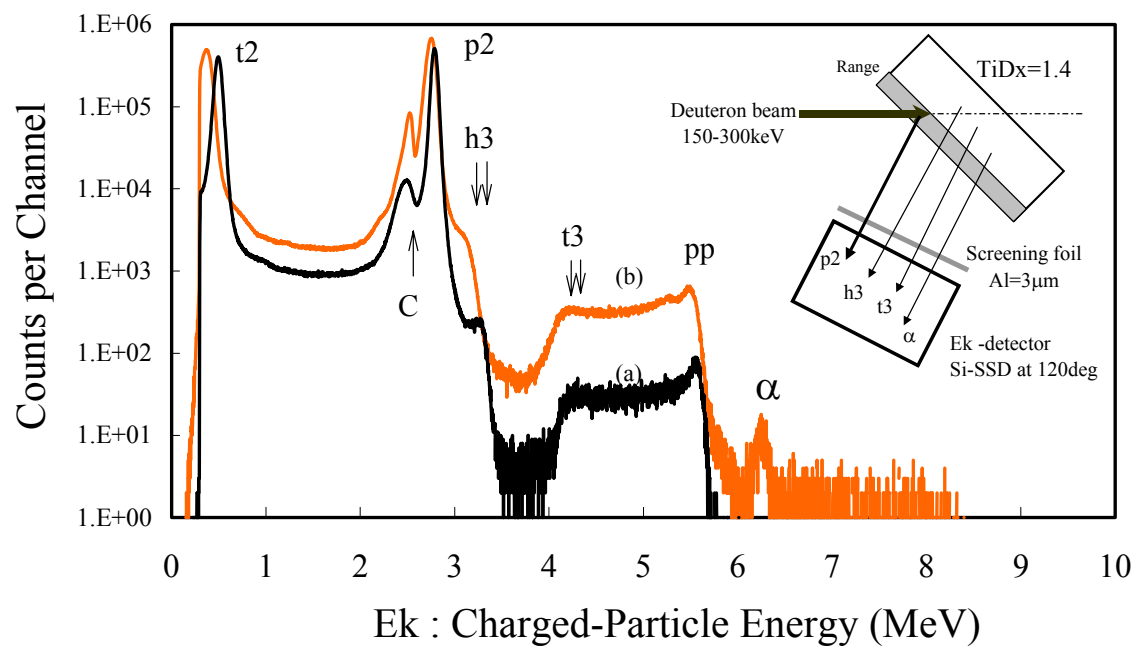

Figure 2. Charged particle spectra detected by Ek-detector, for $\mathrm{D}^{+}$beam energies of $150 \mathrm{keV}$ (a) and $300 \mathrm{keV}$ (b). Peaks at $\mathrm{t} 2$ and $\mathrm{p} 2$ show tritons and protons by 2D fusion. The peak by pp denotes pile-up peak by $\mathrm{p} 2$ protons.

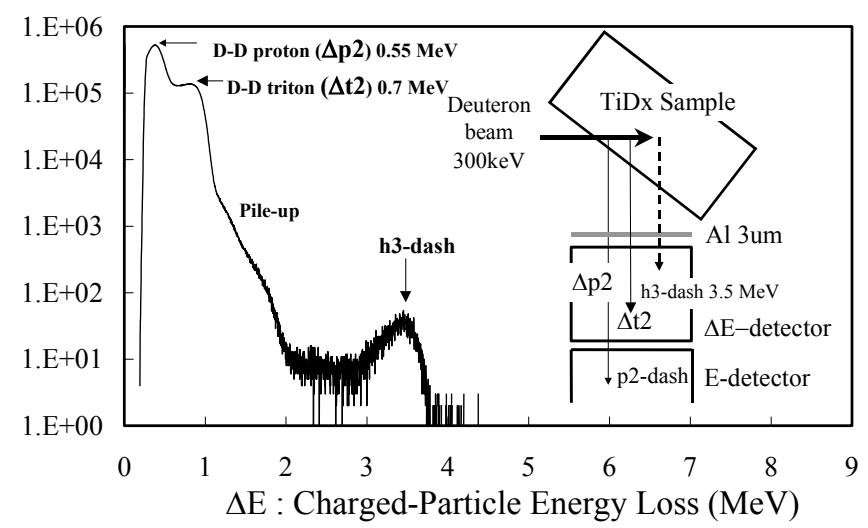

Figure 3. Energy-loss spectra obtained by delta-E detector. Peak h3-dash may correspond to ${ }^{3} \mathrm{He}$ counts by 3D fusion. 


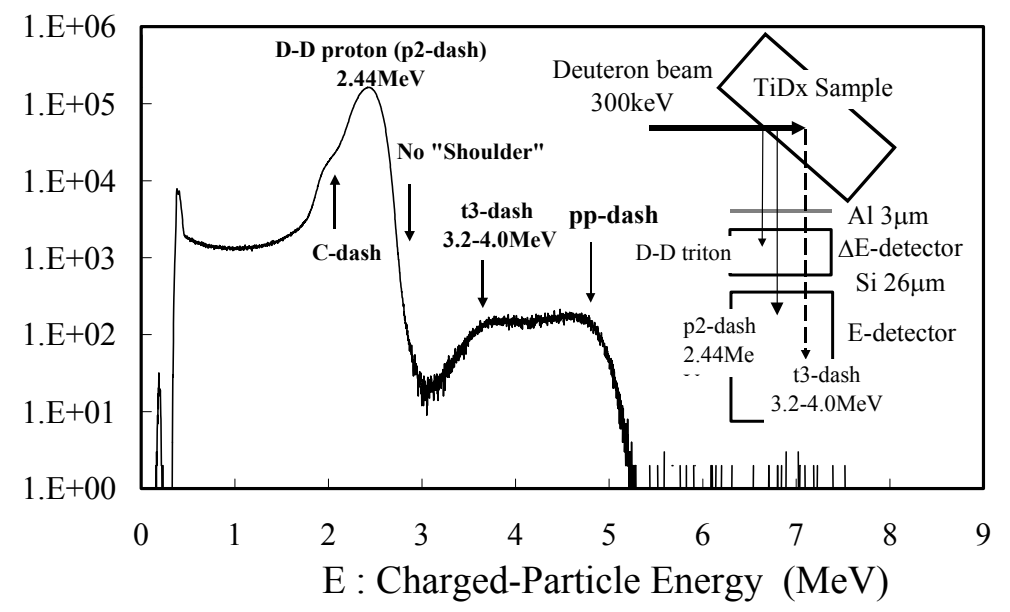

Figure 4. Spectra obtained by E-detector. Shoulder at t3-dash may be contribution by tritons by 3D fusion.

Figure 2 shows the charged particle spectrum emitted from $\mathrm{TiD}_{\mathrm{x}}$ irradiated by $300 \mathrm{keV}$ and $150 \mathrm{keV}$ deuteron beam. Two unique charged particles may have been observed as shoulders marked h3 and t 3 . We suppose they are ${ }^{3} \mathrm{He}(4.75 \mathrm{MeV})$ and $\mathrm{t}(4.75 \mathrm{MeV})$ emitted by $3 \mathrm{D}$ fusion. However, their yield could not be evaluated precisely because the responses of ${ }^{3} \mathrm{He}(4.75 \mathrm{MeV})$ were on the shoulder of D-D protons and the responses of $\mathrm{t}(4.75 \mathrm{MeV})$ were on the pileup signals of D-D protons. To inspect ${ }^{3} \mathrm{He}(4.75 \mathrm{MeV})$, an experiment using a $\Delta \mathrm{E}-\mathrm{E}$ counter telescope was performed. Helium-3 by $3 \mathrm{D}$ fusion stops in the $\Delta \mathrm{E}$ detector and does not reach to $\mathrm{E}$ detector. Figure 3 shows an example of charged particle spectra of $\Delta \mathrm{E}$ detector. The peak measured at about $3.5 \mathrm{MeV}$ in spectrum of the $\Delta \mathrm{E}$ detector was assigned to be ${ }^{3} \mathrm{He}$ counts by $3 \mathrm{D}$ fusion. The ratio of the yield of ${ }^{3} \mathrm{He}$ to that of D-D proton was about $2 \times 10^{-4}$.

In Figure 4, example of spectra taken by E-detector is shown. The shoulder (t3-dash) in 3.2-4 MeV region might be the contribution by tritons of $3 \mathrm{D}$ fusion reactions, although identification is difficult due to overlapping with pile-up signals of 2D protons (pp-dash).

We have tried ${ }^{7}$ measurements with the pile-up reduction technique which was a type of pulse shape separation of pile-up signals from single-event signals. In Figure 5, we show a result. The bump in 4-5 MeV region that remained in the spectrum after pile-up reduction can be identified as the component of tritons $(4.75$ $\mathrm{MeV}$ ) by $3 \mathrm{D}$ fusion. We obtained a similar yield ratio for this $\mathrm{t} 3$-bump and $2 \mathrm{D}$ protons as $2.2 \times 10^{-4}$, which is close to $2 \times 10^{-4}$ for h3-dash in Figure 3. This may indicate that the origin of h3-dash and t3-bump came from same reactions, i.e., branch (b) of 3D fusion, considering the error margin was about $10 \%$.

In Figure 6, we show the beam-energy-dependence of h3-dash yields in delta-E detector spectra for $\mathrm{D}^{+}$ energy of $50 \mathrm{keV}$ to $300 \mathrm{keV}$. For $300 \mathrm{keV}$, h3-dash region may contain impurity reaction by ${ }^{14} \mathrm{~N}(\mathrm{~d}$,alpha) as higher energy-side sub-peak. Contamination by this reaction was only seen at $300 \mathrm{keV}$ and had no significant effect at lower energies, since this impurity reaction has high Coulomb barrier. We have obtained integrated counts in h3-dash regions to get yields for ${ }^{3} \mathrm{He}$ counts for $3 \mathrm{D}$ fusion. 


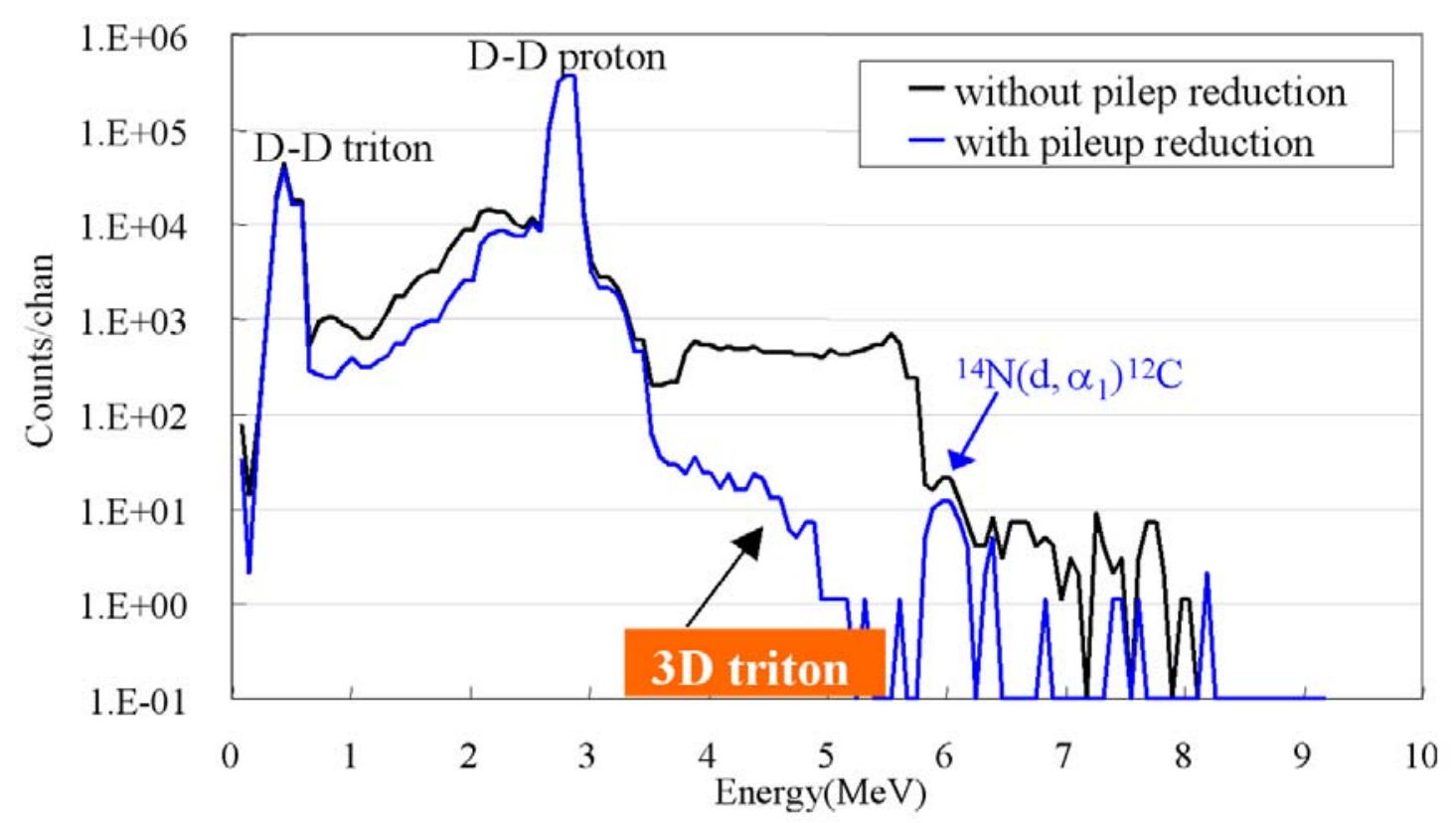

Figure 5. Spectra of Ek detector, before and after the pile-up reduction applied. Bump in 4-5 MeV region after application of pile-up reduction can be tritons by $3 \mathrm{D}$ fusion

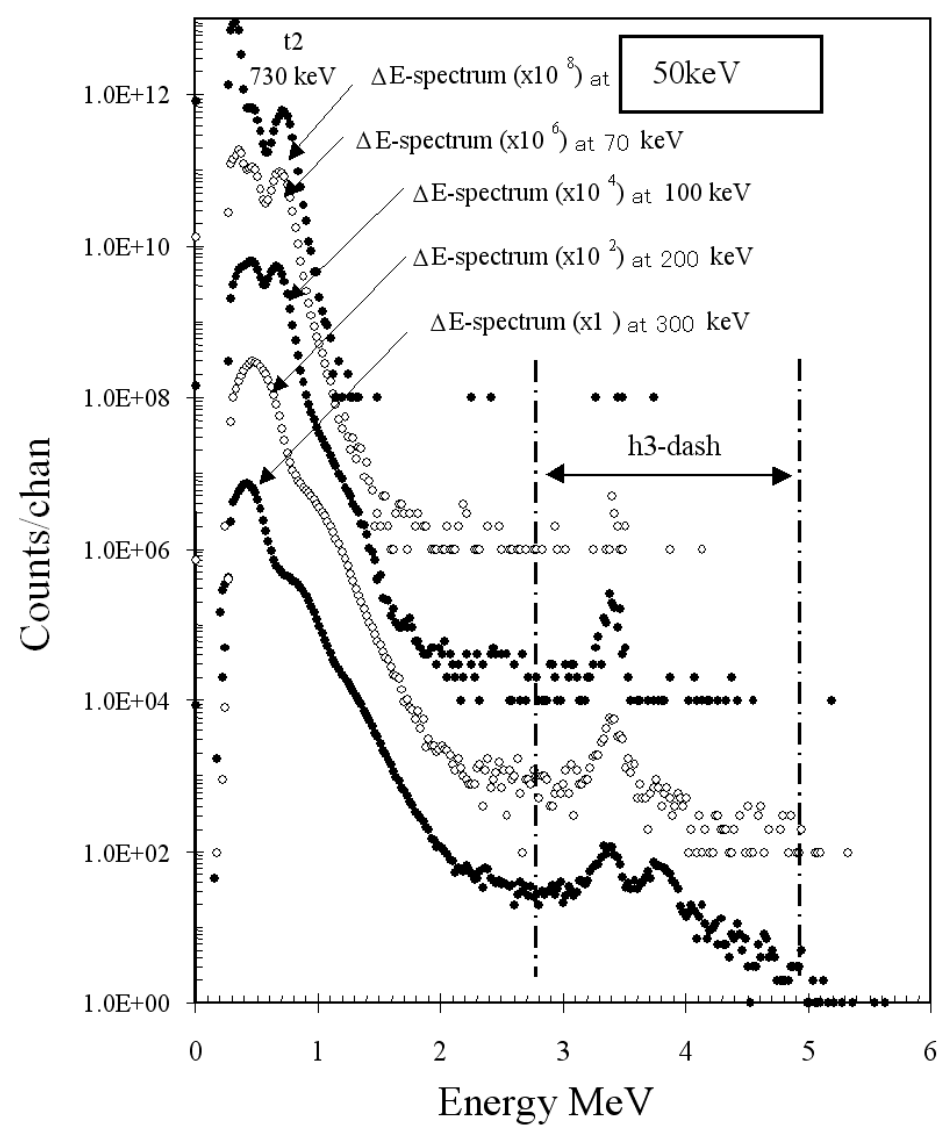

Figure 6. Beam-energy-dependence data of delta-E detector spectra 
Yield ratios between [3D] and [2D] fusion are drawn in Figure 7 as a function of $\mathrm{D}^{+}$beam energy.

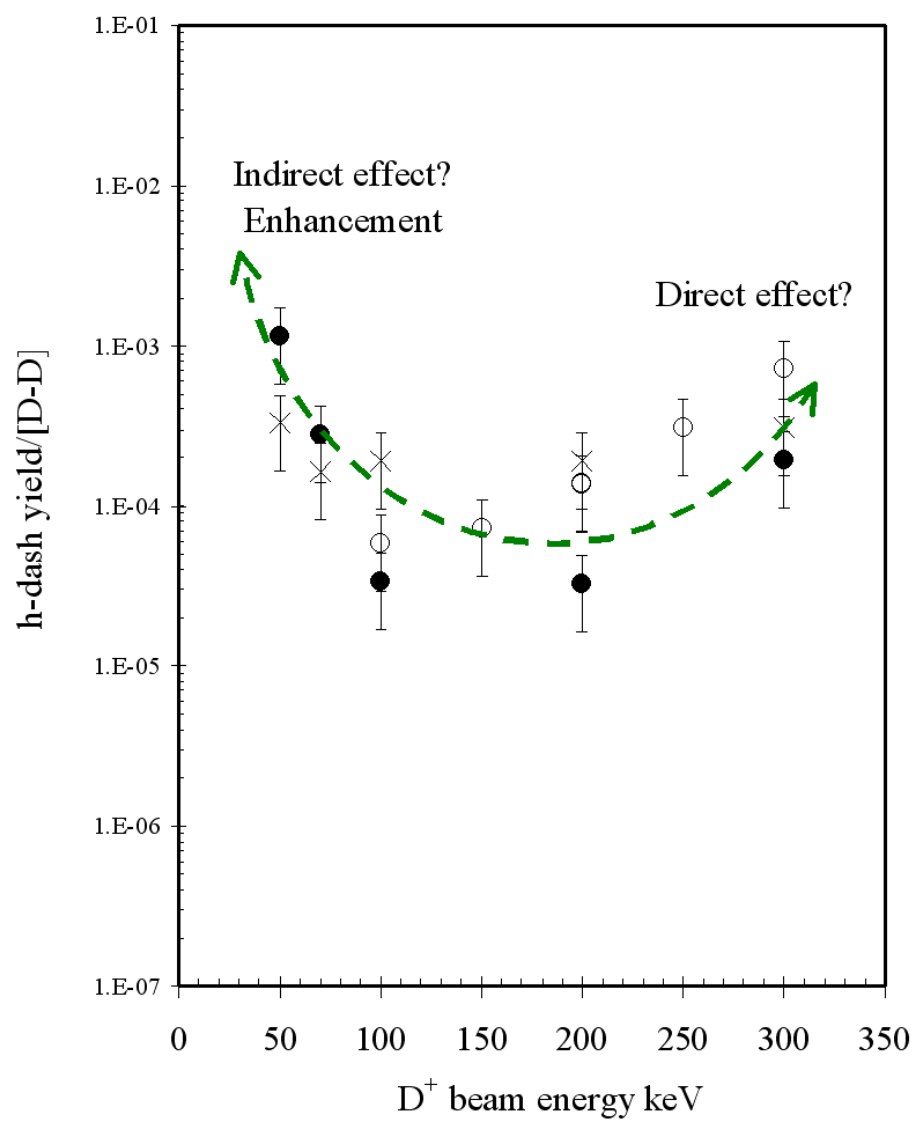

Figure 7. Data of yield ratio $[3 \mathrm{D}] /[2 \mathrm{D}]$ as a function of $\mathrm{D}^{+}$beam energy

The observed yield ratios of $[3 \mathrm{D}] /[2 \mathrm{D}]$ are in the order of $1 \mathrm{E}-3$ to $1 \mathrm{E}-4$. The theoretical estimation of the ratio by the random reaction model (conventional nuclear physics theory) ${ }^{3}$ has given $[3 \mathrm{D}] /[2 \mathrm{D}]$ to be on the order of 1E-30. Our experimental results have shown a drastic enhancement, on the order of $1 \mathrm{E}+25$ to 26 . To explain this by beam-target interaction, we have to assume that $1 \mathrm{E} 12$ close-d-d pairs-in-pm-domain in a cubic cm. If it is so, we have an increasing trend of $[3 \mathrm{D}] /[2 \mathrm{D}]$ ratio in higher $\mathrm{D}^{+}$beam energy because of linearly proportional cascade knock-on reactions during the beam slowing down process in the target sample. The EQPET model ${ }^{5,6}$ predicts that a transient dde* $(2,2)$ state with few pm inter-nuclear distance may be excited with a high density. It may be as high as 1E12 or more by stimulation of D beam implantation into TiDx lattice. However, we see the $[3 \mathrm{D}] /[2 \mathrm{D}]$ ratio is increasing in the lower energy region (about $100 \mathrm{keV}$ ). To explain this we need to consider the occurrence of 3D fusion reactions out of the range of $\mathrm{D}^{+}$beam slowing down. This means that the indirect 3D fusion in lattice was induced by beam stimulation. In this respect, the data is very interesting, and it suggests some sort of "coherent" reaction mechanism.

\section{Alpha $\left({ }^{4} \mathrm{He}\right)+$ D Branch}

We tried to detect high energy (15.9 MeV) deuterons from branch (a) of 3D fusion.

In the experimental system shown in Figure 1, we have a screening (or absorption) foil set up between beamtarget sample and delta-E detector. Usually an Al-foil several micrometers thick is used to prevent scattered deuteron beam from being detected by delta-E detector. In this series of experiments, we changed the screening foil to a very thick (500 micrometer) Ti foil, which absorbed $10.9 \mathrm{MeV}$ of the incident $15.9 \mathrm{MeV}$ deuterons from branch (a) of 3D fusion. With this thick screen in place, $4.9 \mathrm{MeV}$ deuterons come into the rather thick delta-E detector (where 66.5 micrometers of the depletion zone was used). They lost $1.5 \mathrm{MeV}$, and entered the E-detector to deposit the remained $3.2 \mathrm{MeV}$. We make a coincidence measurement for delta-E and E-detector spectra in two-dimensional form. The result from this experiment is shown in Figure 8. 


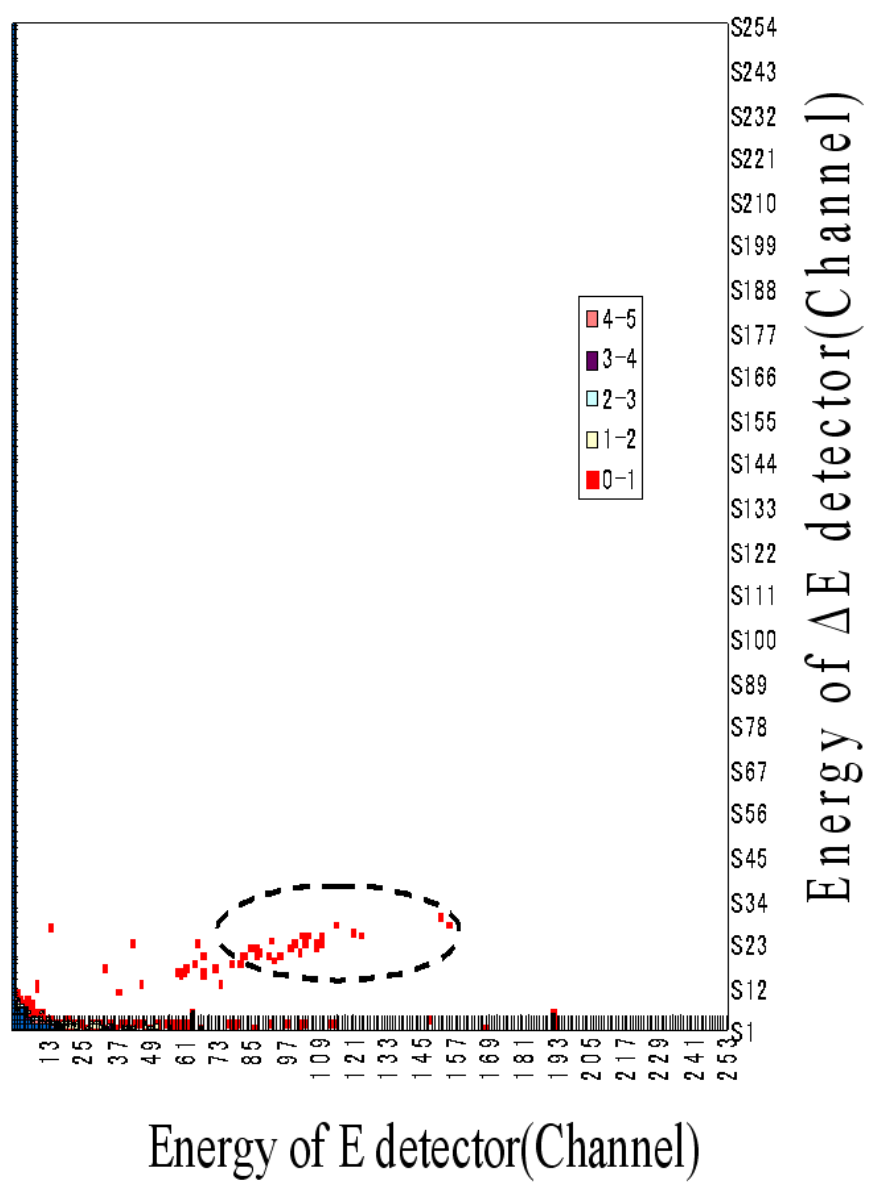

Figure 8. Coincidence spectrum of delta-E and E-detectors, to detect 15.9 MeV deuterons by 3D fusion. The area within broken line of ellipse corresponds to $15.9 \mathrm{MeV}$ deuterons

We observed meaningful counts, as shown in Figure 8, within the expected area of two-dimensional count distribution. Considering the straggling energy spread of deuterons in the 500 micrometers thick Ti screening foil, we could define these signals as $15.9 \mathrm{MeV}$ deuterons by branch (a) of 3D fusion. The yield ratio to $3 \mathrm{MeV}$ protons by $2 \mathrm{D}$ fusion was obtained as $[3 \mathrm{D}] /[2 \mathrm{D}]=2.2 \times 10^{-4}$. This value was very close to the ratio of the ${ }^{3} \mathrm{He}+\mathrm{t}$ branch. So, we conclude that branching ratio for (a) and (b) channels is about one to one.

We have also tried to detect $d$ and alpha of branch (a) with coincidence technique, by using a very thin (a few microns) TiDx sample and detection from the front and back sides simultaneously. We also tried to detect $t$ and ${ }^{3} \mathrm{He}$ by branch (b) in coincidence. The results are that we could not find any meaningful coincidence signals in the expected energy region of two-dimensional maps. We have discussed the probable reason; that the temperature of thin target foil rose too high to keep D in lattice under beam irradiation, since cooling was not effective. Only when we used 0.5 to $1 \mathrm{~mm}$ thick TiDx (x.1.8) samples with Peltier cooling device, could we observe meaningful signals to relate to $3 \mathrm{D}$ fusion for the whole runs in the present study.

\section{H + D + D Reaction and Si Beam Experiment}

Using an $\mathrm{H}^{+}$beam, we have tried ${ }^{4}$ to detect $19 \mathrm{MeV}$ protons by $\mathrm{H}+\mathrm{D}+\mathrm{D}$ fusion. We were able to identify meaningful counts of these high energy protons. This experiment suggests that we should have closely-packed d-d pairs with few pm inter-nuclear distance with certain density (around 1E12 pairs/cc or more), as already discussed for $\mathrm{D}^{+}$beam experiment. 
To search for better evidence of indirect 3D fusion, Si beam (2 MeV) irradiation into TiDx sample was done ${ }^{7}$. We found several counts in 3-4 MeV region, which we believe is positive evidence for the occurrence of indirect 3D fusion (true "cold" fusion).

\section{Conclusions}

We have done a series of experiments over twelve years trying to detect the products of 3D fusion in condensed matter (metal-deuterides) under beam irradiation, as evidence of the existence of highly enhanced density of closely packed d-d pairs stimulated in condensed matter. We conclude that we have definitely seen positive evidence of drastically enhanced 3D fusion in condensed matter under stimulation of beam irradiation.

\section{References}

1) A. Takahashi, et al: Fusion Technology, Vol.27 (1995) pp.71-85

2) A. Takahashi, et al: Physics Letters A 255 (1999) 89-97

3) A. Takahashi, et al: Fusion Technology, Vol.34 (1998) pp.256-272

4) Y. Isobe, et al: Jpn. J. Appl. Phys. Vol.41 (2002) pp.1546-1556

5) A. Takahashi: Proc. JCF4, October 2002, Iwate Japan, pp.74-78 (proceedings of JCF4 is available at http://www.eng.osaka-u.ac.jp/nuc/03/nuc03web/JCF/)

6) A. Takahashi: Mechanism of deuteron cluster fusion by EQPET model, this conference

7) T. Dairaku, et al.: Proc. ICCF9, Beijing China 2002, 73 (2002) 